\title{
Adaptive metabolic changes in CADASIL white matter
}

\section{Journal Article}

\section{Author(s):}

Akhvlediani, Tamar; Henning, Anke; Sandor, Peter S.; Bösiger, Peter; Jung, Hans H.

Publication date:

2010

Permanent link:

https://doi.org/10.3929/ethz-b-000017222

Rights / license:

In Copyright - Non-Commercial Use Permitted

Originally published in:

Journal of neurology 257(2), https://doi.org/10.1007/s00415-009-5281-5 


\title{
Adaptive metabolic changes in CADASIL white matter
}

\author{
Tamar Akhvlediani · Anke Henning • \\ Peter S. Sándor · Peter Boesiger · Hans H. Jung
}

Received: 9 December 2008/Revised: 3 July 2009/Accepted: 4 August 2009/Published online: 19 August 2009

(C) Springer-Verlag 2009

\begin{abstract}
Cerebral autosomal dominant arteriopathy with subcortical infarcts and leukoencephalopathy (CADASIL) is an important genetic cause of stroke, but pathogenic mechanisms and functional alterations remain poorly characterized. The purpose of this study was to investigate adaptive metabolic and functional changes in white matter hyperintensities and normal-appearing white matter in CADASIL patients using ${ }^{1} \mathrm{H}$-magnetic resonance spectroscopic imaging (MRSI). Eight CADASIL patients and eight matched healthy controls were studied. ${ }^{1} \mathrm{H}-\mathrm{MRSI}$ data were acquired on a $3 \mathrm{~T}$ scanner using high-resolution multi-spin echo spectroscopic imaging $\left(T_{\mathrm{E}}=288 \mathrm{~ms}\right)$ and non-accelerated medium-resolution MRSI $\left(T_{\mathrm{E}}=35 \mathrm{~ms}\right)$. MRI of all CADASIL patients demonstrated characteristic white matter hyper-intensities (WMH) in the subcortical periventricular white matter. Cre/Cho, Glx/Cho and Glx/Cre ratios were significantly decreased in WMH compared to normal-appearing white matter (NAWM) in patients, while Glx/Cre and $\mathrm{mI} / \mathrm{Cho}$ ratios in NAWM showed a significant increase compared to healthy controls. In severely affected patients derived spectra reflected a decrease of NAA concentrations inside WMH when compared to healthy white matter. Metabolic abnormalities in WMH of CADASIL patients are compatible with axonal loss due to chronic micro-infarctions. Increased Glx/Cre and
\end{abstract}

T. Akhvlediani and A. Henning contributed equally.

T. Akhvlediani · P. S. Sándor · H. H. Jung ( $\)$

Department of Neurology, University Hospital Zürich,

Frauenklinikstrasse 26, 8091 Zurich, Switzerland

e-mail: hans.jung@usz.ch

T. Akhvlediani $\cdot$ A. Henning $\cdot$ P. Boesiger

Institute for Biomedical Engineering,

University and Swiss Federal Institute

of Technology Zürich, Zurich, Switzerland
$\mathrm{mI} /$ Cho ratios in NAWM indicate an augmented glial cell density and decreased neuronal cell density. This altered tissue composition might be interpreted as adaptation to hypoperfusion and impaired vasoreactivity in NAWM of CADASIL patients. Our data might contribute to the general understanding of adaptive processes induced by hypoperfusion and chronic ischemia.

Keywords CADASIL - Magnetic resonance spectroscopy $\cdot$ fMRI $\cdot$ MRSI

\section{Introduction}

Cerebral autosomal dominant arteriopathy with subcortical infarcts and leukoencephalopathy (CADASIL) is an inherited cerebral small vessel disease caused by mutations in the Notch3 gene [8, 16, 29]. Clinical manifestations include migraine with aura, transient ischemic attacks, recurrent strokes, psychiatric disorders, and progressive cognitive impairment with an important variability of clinical presentation $[10,25,26]$. CADASIL has been recognized as the most common cause of hereditary stroke, but probably remains still under-diagnosed [8,9]. Diagnosis of CADASIL is confirmed by the presence of granular osmiophilic materials in smooth muscle cells of the skin and muscle vessels $[16,27]$ or molecular genetic analysis of the Notch3 gene [14]. Since the underlying mechanism of CADASIL is a progressive degeneration of the smooth muscle cells in blood vessels, an increasing impairment of adaptive vasodilation, especially of the small brain arteries, might be present. Indeed, previous SPECT and PET studies have demonstrated hypoperfusion and decreased vasoreactivity $[5,21,23]$. Hence we hypothesize that, in addition to metabolic changes due to 
the stroke episodes and micro infarctions, the metabolism of unaffected brain tissue might also adapt to the hypoperfusive state.

Cerebral T2-weighted and FLAIR MRI in CADASIL typically shows confluent white matter signal hyperintensities with characteristic involvement of external capsules and anterior temporal poles [20]. Previous proton magnetic resonance spectroscopy studies mainly focused on these hyper-intense white matter lesions and demonstrated metabolic abnormalities that suggested axonal injury, enlarged extracellular spaces, myelin loss, and gliosis due to microinfarctions and stroke episodes [2, 17, 19, 22]. However, results were partly inconsistent concerning changes in normal-appearing white matter, and the pathogenic mechanisms and the evolution of the alterations remain poorly characterized.

The present study focused on the characterization of differential metabolic alterations in affected and normalappearing white matter, possibly indicating adaptive changes in the unaffected white matter of CADASIL patients due to hypoperfusion, thus aiming to shed light on disease progression and ischemic preconditioning. A refined protocol based on medium-resolution short-echo (to assess the spatial distribution of an enlarged number of metabolites and to avoid misleading $\mathrm{T}_{2}$ relaxation effects) and high-resolution long-echo (to assess the spatial distribution of lactate and to achieve good correspondence of metabolite maps and anatomical reference) proton MR spectroscopic imaging (to compare metabolic changes in affected and normal-appearing periventricular white matter of CADASIL patients) was used. The investigated metabolites were $N$-acetyl-aspartate (NAA) - a marker for neuronal density and function, myo-inositol (mI) - an astro-glial marker, glutamate plus glutamine (Glx)—-markers for energy metabolism and astro-glial neurotransmitter cycling [30], choline containing compounds-a marker of alterations in membrane synthesis as well as the sum of phosphocreatine and dephosphorylated creatine (Cre)-which represent an energy buffer system and lactate (Lac) - a marker for increased anaerobic glycolysis [7].

\section{Patients and methods}

Patients and volunteers

We examined 8 CADASIL patients from 6 unrelated families ( 3 males and 5 females; mean age 53 years, range 41-68) and 8 age and sex matched healthy volunteers (3 males and 5 females; mean age 50 years, range 41-63). The study was approved by the local ethics committee, and all probands gave written informed consent. Diagnosis of CADASIL was confirmed in all patients by skin biopsy and/or molecular genetic analysis. The patients underwent a structured interview and a neurological examination. Clinical characteristics are summarized in Table 1. Four patients had migraine with aura preceding other CADASIL manifestations, one patient had isolated migraine auras without headaches, and three patients had no migraine. Four patients had sudden, transient focal neurological disturbances without spreading, suspicious for transient ischemic attacks, and two patients had strokes. One patient had repetitive attacks of dizziness interpreted as vestibular migraine. One patient had cognitive decline, depression and a complex dyskinetic movement disorder. One patient had a right-dominant extrapyramidal syndrome. None of

Table 1 Clinical data and genetic analysis

\begin{tabular}{|c|c|c|c|c|c|}
\hline Case & Gender & $\begin{array}{l}\text { Age at } \\
\text { examination }\end{array}$ & $\begin{array}{l}\text { Notch3 } \\
\text { mutation }\end{array}$ & Clinical features & Migraine type \\
\hline 1 & M & 60 & $\mathrm{~A} 133 \mathrm{C}$ & Single TIA with speech arrest & None \\
\hline 2 & $\mathrm{~F}$ & 68 & $\mathrm{C} 174 \mathrm{~T}$ & $\begin{array}{l}\text { Repetitive episodes of dizziness } \\
\text { Sensory aura without migraine }\end{array}$ & Aura without migraine \\
\hline 3 & $\mathrm{~F}$ & 41 & $\mathrm{C} 174 \mathrm{~T}$ & Migraine with sensory and visual aura & Migraine with aura \\
\hline 4 & $\mathrm{~F}$ & 47 & $\mathrm{R} 184 \mathrm{C}$ & $\begin{array}{l}\text { TIA with hemihypesthesia } \\
\text { Migraine with sensory and visual aura }\end{array}$ & Migraine with aura \\
\hline 5 & $\mathrm{~F}$ & 64 & $\mathrm{R} 169 \mathrm{C}$ & $\begin{array}{l}\text { Depression, repetitive strokes, subcortical dementia, } \\
\text { focal epilepsy, generalized dyskinesia, } \\
\text { migraine with sensory and visual aura }\end{array}$ & Migraine with aura \\
\hline 6 & $\mathrm{~F}$ & 46 & $\mathrm{R} 169 \mathrm{C}$ & $\begin{array}{l}\text { TIA with brainstem symptoms } \\
\text { Migraine with sensory and visual aura }\end{array}$ & Migraine with aura \\
\hline 7 & M & 41 & $\mathrm{C} 65 \mathrm{~T}$ & Repetitive strokes with hemiparesis and aphasia & None \\
\hline 8 & M & 58 & $\mathrm{R} 182 \mathrm{C}$ & $\begin{array}{l}\text { TIA with hemianopsia } \\
\text { Right-dominant rigidity }\end{array}$ & None \\
\hline
\end{tabular}

The skin biopsy was positive in all patients 
the patients had a stroke or migraine episode within a month before the participation in the study. The structural MRI images were typical for CADASIL in all patients with confluating subcortical white matter lesions including the anterior temporal pole.

Magnetic resonance imaging

All MR measurements were performed on a three Tesla whole body scanner (Philips Achieva, Philips Medical Systems, Best, The Netherlands) using a Philips transmit/ receive head coil. Subjects underwent combined brain MRI/MRSI examination. Anatomical $\mathrm{T}_{2}$-weighted turbo spin echo images (sagittal, coronal, transversal) were acquired to assess the white matter lesions and to plan the volumes of interest for MRSI.

\section{Magnetic resonance spectroscopy}

${ }^{1} \mathrm{H}-\mathrm{MRSI}$ data were acquired using multi spin-echo spectroscopic imaging (turbo spectroscopic imaging, TSI) and unaccelerated MRSI using a sampled bandwidth of $2,500 \mathrm{~Hz}$ and a repetition time of $1,520 \mathrm{~ms}$. Signal localization was achieved by use of over-prescribed pointresolved spectroscopy in combination with a $T_{1}$ and $B_{1}$ insensitive outer volume suppression scheme based on broadband saturation pulses with polynomial phase response [13]. Chemical shift selective spectroscopy [12] was used for water suppression.

Long-echo time multi-spin echo spectroscopic imaging (acceleration factor 3 ) enabled a high spatial resolution $\left(T_{\mathrm{E}}=288 \mathrm{~ms}\right.$, voxel size $\left.=8 \times 8 \times 15 \mathrm{~mm}\right)$ at a short measurement time $(6 \mathrm{~min})$ and was used to compare affected and normal-appearing white matter of CADASIL patients with healthy periventricular white matter in volunteers. Unaccelerated short echo-time MRSI with a medium spatial resolution $\left(T_{\mathrm{E}}=35 \mathrm{~ms}\right.$, voxel size $=$ $14 \times 14 \times 20 \mathrm{~mm}$ ) enabled detection and evaluation of additional metabolites, namely $\mathrm{mI}$ and Glx. Data were obtained from a transversal slice aligned along the edge of the corpus callosum and adjusted to cover occipital periventricular white matter (Fig. 1). To ensure that white matter lesions, normal-appearing white matter or healthy periventricular white matter would fill the entire slice thickness at certain positions in order to avoid throughslice partial volume effects, planning of the MRSI examination was supported by sagittal and coronal $\mathrm{T} 2$-weighted scans (Fig. 1a).

The custom written software SIview (Institute for Biomedical Engineering, University and ETH Zurich, Switzerland) was used to visualize spectra and metabolite maps. Spectral data were post-processed using a constant baseline
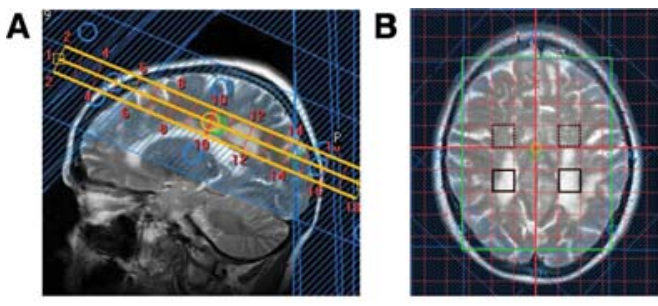

E

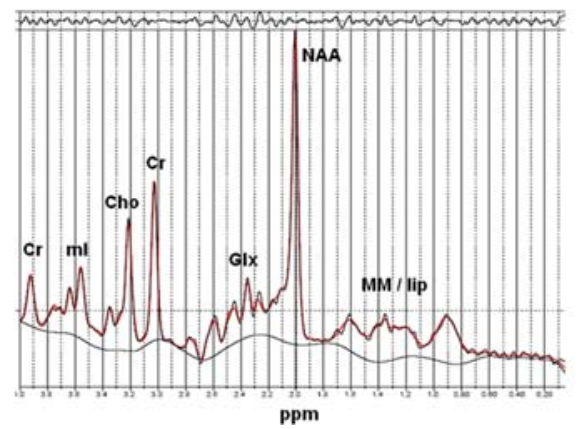

Fig. 1 MRSI planning and data quality. Typical positions of the MRSI grid (red), the MRSI slice (orange), the PRESS volume (green) and the OVS bands (blue) (a-d) as well as averaged voxels inside white matter lesion (black, b, c), patient NAWM (black/dotted, b, c) and white matter in $\mathrm{HC}$ (white, d). In patients and healthy volunteers MRSI slices were aligned to the corpus callosum and included as much posterior periventricular white matter as possible $(\mathbf{a}-\mathbf{d})$. The chosen slice thickness and position as well as selected voxel positions
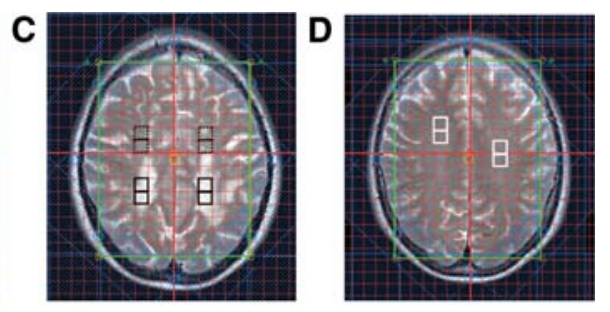

$\mathbf{F}$

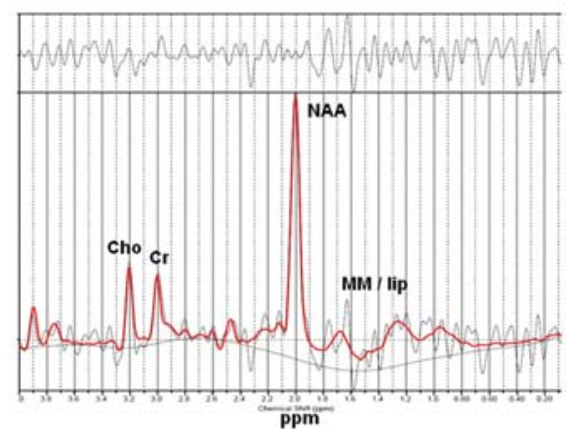

inside the slice ensured that periventricular WMH and NAWM in patients $(\mathbf{a}-\mathbf{c})$ as well as periventricular white matter in $\mathrm{HC}(\mathbf{d})$ filled out certain voxels as completely as possible throughout the entire slice in order to avoid partial volume effects. Typical spectral quality of non-apodized average spectra and corresponding LC-Model fits for short-echo time MRSI ( $T_{\mathrm{E}}=35 \mathrm{~ms}, 2$ averaged voxels each) (e) and long-echo time TSI $\left(T_{\mathrm{E}}=288 \mathrm{~ms}, 4\right.$ averaged voxels each) (f) demonstrate reliable quantification 
correction, $\mathrm{B}_{0}$-correction, exponential spectral filtering by $3 \mathrm{~Hz}$, as well as manual zero-order and linear phase correction.

Metabolite concentrations were estimated using LC Model [24] from non-apodized average spectra. For the TSI and short-echo MRSI data sets the average spectra either contained only voxels inside WMH or the same number of voxels inside NAWM in patients or corresponding healthy white matter in controls, respectively (Fig. 1). Selection for regions of interest in patients depended on the location of white matter lesions inside occipital, periventricular white matter. A voxel was considered a "WMH"-voxel if it mainly contained hyperintense white matter and a "NAWM"-voxel if it mainly contained normal-appearing white matter with maximally $10 \%$ contributions of other tissue types. Voxel content was inspected visually based on a T2-weighted TSE examination that had the same orientation and off-center as the MRSI slice; the area covered by the MRSI slice was subdivided into five imaging slices.

Four averaged voxels were used for each tissue type for the high-resolution TSI data sets and two averaged voxels for the medium-resolution MRSI dataset (Fig. 1). Considering the echo times of $T_{\mathrm{E}}=35 \mathrm{~ms}$ and $T_{\mathrm{E}}=288 \mathrm{~ms}$, two sets of phantom spectra of 19 individual brain metabolites each were used as a metabolite basis for fitting the LC Model [24] to the in vivo spectra.

A widely used approach in spectroscopy is to quantify resonance lines in terms of metabolite ratios, i.e. ratios of intensities of particular resonance signals. This approach corrects for many factors that otherwise influence quantification results such as $B_{1}$ inhomogeneity, coil load changes and changing receive gain settings. The signal arising from Cre is most frequently taken as an internal reference since it remains stable during neuronal activation. Thus NAA/Cre, mI/Cre, Glx/Cre and Cho/Cre ratios were calculated from the LC Model fitting results expressed in institutional units (IU). Due to the possible distortion of energy metabolism in CADASIL patients, we additionally used Cho as an internal reference, since this is stable as long as no major structural changes occur. Thus, NAA/ Cho, $\mathrm{mI} / \mathrm{Cho}$ and Glx/Cho ratios were also calculated. The short-echo MRSI data of one subject were not quantifiable due to pronounced movement artefacts which affected the quality of the spectra.

\section{Statistics}

Data were analyzed for each subject individually as described above. To account for multiple comparisons among multiple subgroups, a repeated-measures analysisof-variance (repeated-measures ANOVA) was performed comparing all metabolites and metabolite ratios between
Table 2 Relative metabolite concentrations \pm SEM from TSI data: $T_{\mathrm{E}}=288 \mathrm{~ms}$, voxel size $=8 \times 8 \times 15 \mathrm{~mm}$

\begin{tabular}{lllll}
\hline & WMH & NAWM & HC & ALL \\
\hline NAA/Cho & $10.28 \pm 1.55$ & $10.75 \pm 1.91$ & $13.474 \pm 3.13$ & n.s. \\
NAA/Cre & $5.06 \pm 0.52$ & $4.70 \pm 0.95$ & $4.82 \pm 0.89$ & n.s. \\
Cre/Cho & $2.36 \pm 0.33$ & $2.87 \pm 0.55$ & $2.96 \pm 0.39$ & $F=3.605$ \\
& $* p=0.069$ & $* p=0.069$ & & $p<0.066$ \\
\hline
\end{tabular}

Significant differences ( $p<0.05$; uncorrected) between all subgroups (last column) and between WMH and NAWM (*) are indicated

white matter hyper-intensities (WMH), normal-appearing white matter in patients (NAWM) and white matter in healthy controls (HC) for the TSI data as well as for the short-echo MRSI data sets. Subsequently, multiple univariate $F$ test based ANOVAs were performed for each metabolite ratio to reveal their individual contributions to the subgroup differences found in the first analysis step (see columns labeled "ALL" in Tables 2, 3). Two-tailed, paired Student's $t$ tests were used post hoc to compare metabolite levels in between the subgroups. $p$ values $<0.05$ were considered as statistically significant and $p$ values $<0.1$ as a statistical trend. Due to the small sample size, normal distribution was assumed and the Student's $t$ test was used in preference to parametric tests. The statistical analysis was performed using SPSS 15.0.1. (SPPS inc., www.spss.com).

\section{Results}

Anatomical imaging

MRI of all CADASIL patients demonstrated characteristic $\mathrm{WMH}$ in the subcortical periventricular white matter as well as in the temporal poles. By visual inspection, the lesions tended to be more pronounced in older patients even if only mild clinical manifestations were present. These changes indicate that $T_{2}$ relaxation times underlie significant changes found when comparing white matter lesions and normal-appearing white matter of CADASIL patients.

\section{General group differences}

Repeated-measures ANOVA analysis revealed significant general group differences between WMH, NAWM and HC based on the long-echo time high-resolution TSI data $(F=1.847 ; p=0.039)$ as well as on the short-echo time low resolution data $(F=7.689 ; p=0.007)$. For the TSI data three metabolite ratios were considered, while for the short-echo MRSI data seven metabolite ratios were included in this analysis (Tables 2, 3). 
Relative metabolite concentrations

Univariate ANOVA analyses performed based on results from high-resolution long-echo TSI measurements indicated general group differences between WMH, NAWM and $\mathrm{HC}$ only regarding the Cre/Cho ratio (Table 2, column "ALL"). Specifically, the Cre/Cho ratio was significantly decreased in WMH compared to NAWM (Table 2, columns "WMH" and "NAWM").

This finding was confirmed by medium-resolution shortecho MRSI measurements. In addition, univariate ANOVA analyses based on the short-echo MRSI data indicated general group differences between WMH, NAWM and HC for $\mathrm{mI} / \mathrm{Cho}$, Glx/Cho and Glx/Cre ratios (Table 3, column "ALL"). The Glx/Cho and Glx/Cre ratios were significantly decreased in WMH compared to NAWM. In addition, Glx/Cre ratios and $\mathrm{mI} / \mathrm{Cho}$ ratios were significantly increased in NAWM compared to HC (Table 3, columns "WMH", "NAWM" and "HC").

The following equations summarize the results:

$$
\begin{aligned}
{[\mathrm{Cre} / \mathrm{Cho}]_{\mathrm{WMH}} } & <[\mathrm{Cre} / \mathrm{Cho}]_{\mathrm{NAWM}} \\
{[\mathrm{Glx} / \mathrm{Cho}]_{\mathrm{WMH}} } & <[\mathrm{Glx} / \mathrm{Cho}]_{\mathrm{NAWM}} \\
{[\mathrm{Glx} / \mathrm{Cre}]_{\mathrm{WMH}} } & <[\mathrm{Glx} / \mathrm{Cre}]_{\mathrm{NAWM}} \\
{[\mathrm{Glx} / \mathrm{Cre}]_{\mathrm{HC}} } & <[\mathrm{Glx} / \mathrm{Cre}]_{\mathrm{NAWM}} \\
{[\mathrm{mI} / \mathrm{Cho}]_{\mathrm{HC}} } & <[\mathrm{mI} / \mathrm{Cho}]_{\mathrm{NAWM}}
\end{aligned}
$$

In CADASIL patients with extended WMH, TSI and MRSI derived average spectra visually reflect a marked decrease of NAA/Cre and NAA/Cho ratios inside WMH compared to healthy white matter (Fig. 2). Group differences are only indicative for altered NAA/Cho ratios but not statistically significant if all patients are included in the group comparison

Table 3 Relative metabolite concentrations \pm SEM from CSI data: $T_{\mathrm{E}}=35 \mathrm{~ms}$, voxel size $=14 \times 14 \times 20 \mathrm{~mm}$

\begin{tabular}{lllll}
\hline & WMH & NAWM & HC & ALL \\
\hline NAA/Cho & $8.37 \pm 1.14$ & $10.30 \pm 0.97$ & $9.58 \pm 1.04$ & n.s. \\
Cre/Cho & $3.32 \pm 0.17$ & $4.74 \pm 0.39$ & $3.74 \pm 0.41$ & $F=4.473$ \\
& $* p=0.016$ & $* p=0.016$ & & $p<0.030$ \\
mI/Cho & $2.10 \pm 0.22$ & $2.66 \pm 0.24$ & $2.02 \pm 0.16$ & $F=2.776$ \\
& & $* * p=0.090$ & $* * p=0.090$ & $p<0.094$ \\
Glx/Cho & $4.77 \pm 0.70$ & $8.17 \pm 1.09$ & $5.25 \pm 0.61$ & $F=4.884$ \\
& $* p=0.094$ & $* p=0.094$ & & $p<0.023$ \\
NAA/Cre & $2.46 \pm 0.30$ & $2.13 \pm 0.07$ & $2.57 \pm 1.34$ & n.s \\
mI/Cre & $0.63 \pm 0.06$ & $0.56 \pm 0.03$ & $0.55 \pm 0.04$ & n.s \\
Glx/Cre & $1.42 \pm 0.15$ & $1.73 \pm 0.18$ & $1.39 \pm 0.08$ & $F=23.543$ \\
& $* p=0.001$ & $* p=0.001$ & $* * p=0.0001$ & $p<0.0001$ \\
& & $* * p=0.0001$ & &
\end{tabular}

Significant differences $(p<0.05$; uncorrected $)$ and trends $(p<0.1$; uncorrected) between all subgroups (last column) and WMH and NAWM $(*)$, or NAWM and HC $(* *)$ are indicated
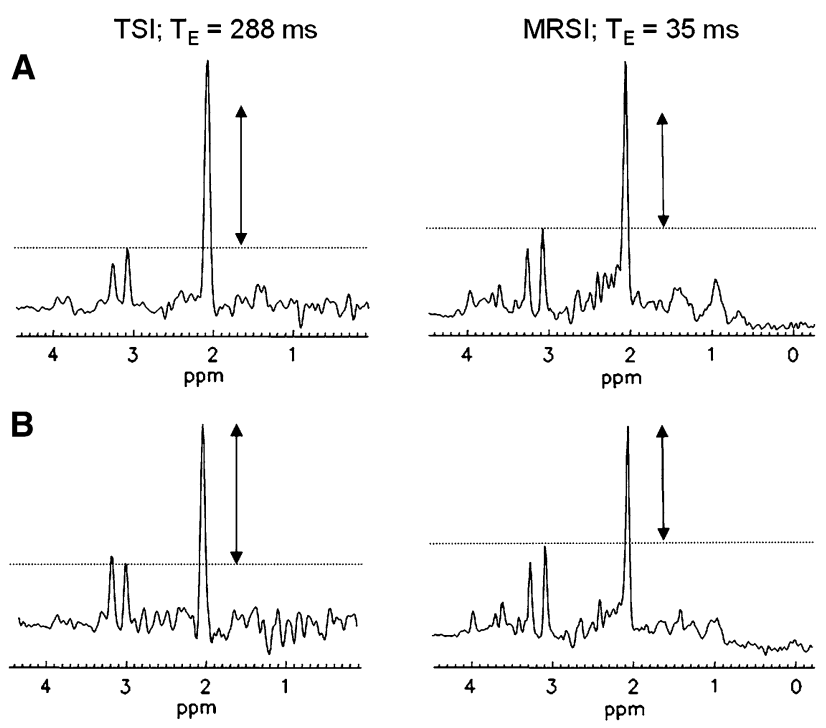

Fig. 2 NAA reduction in CADASIL white matter. Average spectra from white matter of an $\mathrm{HC}$ (a) as well as of a CADASIL patient with extended WMH (b) from corresponding TSI $\left(T_{\mathrm{E}}=288 \mathrm{~ms}\right)$ and MRSI $\left(T_{\mathrm{E}}=35 \mathrm{~ms}\right)$ data sets. The positions of the averaged voxels for the medium-resolution MRSI ( 2 averaged voxels each) and the high-resolution TSI (4 averaged voxels each) are indicated in Fig. 1bd. CADASIL patients with extended WMH (b) showed a marked NAA/Cre and NAA/Cho ratio reduction (arrow sizes are derived from WMH) compared to healthy controls (a)

(Tables 1,2). None of the CADASIL patients or volunteers showed a marked increase in lactate sufficient to reach detectable levels.

\section{Discussion}

The present study was designed to investigate metabolic changes in white matter hyperintensities and normal-appearing white matter in CADASIL patients using ${ }^{1} \mathrm{H}$-MRSI.

Decreased $\mathrm{Cr} / \mathrm{Cho}$, Glx/Cho and Glx/Cre ratios in WMH compared to those in NAWM of CADASIL patients were found, while there were no statistically significant differences between WMH and HC. In addition, Glx/Cre and $\mathrm{mI} / \mathrm{Cho}$ ratios were found to be significantly increased in NAWM of CADASIL patients if compared to healthy periventricular white matter. In line with one previous study [19], but contradicting earlier results $[2,29]$, no statistically significant differences were found for mean NAA/Cho or mean NAA/Cre ratios, neither between WMH and HC nor between WMH and NAWM. This might be explained by substantial variations regarding the neuronal density among the patient population. This is supported by the exclusive observation of an unambiguous decrease of NAA in WMH compared to healthy periventricular white matter in the most severely effected elderly CADASIL patients (Fig. 2).

In accordance with histopathologic data, the observed pattern of metabolite alterations reflects the ischemic nature 
of WMH in CADASIL [4, 11, 15, 28]. Since there was no substantial lactate increase, we have no spectroscopic evidence for acute ischemia in the patients examined. The decrease of NAA in severely affected patients indicates a chronic axonal loss. In addition, a slight increase of membrane turnover and a distortion of the energy metabolism are indicated by decreased mean Cre/Cho and Glx/Cho ratios in WMH. Hence, our results suggest a slowly progressive process due to repetitive micro-infarctions.

In contrast to WMH, NAWM of CADASIL patients shows an increase in Glx/Cre and $\mathrm{mI} / \mathrm{Cho}$ ratios compared to healthy periventricular white matter. These findings are not compatible with chronic or acute ischemia and might, rather, reflect an increased astroglial cell density and a decreased neuronal cell density compared to controls [1, 3]. In accordance with our findings, a reduction of the fractional anisotropy in NAWM was measured by diffusion weighted MR imaging [6]. Although the present study is limited by the lack of absolute quantification and the small sample size, the proposed alterations in neuronal and glial cell numbers might represent a long-term adaptation of NAWM to hypoperfusion and impaired vasoreactivity and, more specifically, to impaired adaptive vasodilatation of the small brain arteries during increased neuronal activation. Hypoperfusion and decreased vasoreactivity have been demonstrated before using SPECT and PET, thus supporting this interpretation $[5,18,21]$.

In summary, metabolic abnormalities in WMH of CADASIL patients are compatible with axonal loss due to chronic micro-infarctions, thus being in line with previous histopathological and MRS studies. Moreover, increased Glx/Cre and mI/Cho ratios in NAWM indicate an augmented glial cell density and decreased neuronal cell density. This altered tissue composition might be interpreted as adaptation to hypoperfusion and impaired vasoreactivity in NAWM of CADASIL patients. Therefore, our data demonstrate differential metabolic and functional alterations in WMH and NAWM of CADASIL brains, which might give insight into adaptive processes of chronic ischemia in general.

Acknowledgments T.A. received a fellowship of the European Neurological Society (ENS). The study was supported by the CADASIL Foundation of America. We thank Thomas Lange and Ulrike Dydak for providing SIview and their advice on the protocol optimization for the lactate detection and the Department of Statistics, Faculty of Medicine, University of Zurich, for statistical support.

Conflicts of interest statement None.

\section{References}

1. Angelie E, Bonmartin A, Boudraa A, Gonnaud PM, Mallet JJ, Sappey-Marinier D (2001) Regional differences and metabolic changes in normal aging of the human brain: proton MR spectroscopic imaging study. Am J Neuroradiol 22:119-127

2. Auer DP, Schirmer T, Heidenreich JO, Herzog J, Puetz B, Dichgans M (2001) Altered white, grey matter metabolism in CADASIL: a proton MR spectroscopy and 1H-MRSI study. Neurology 56:635-642

3. Barkhof F, van Walderveen M (1999) Characterization of tissue damage in multiple sclerosis by nuclear magnetic resonance. Philos Trans R Soc Lond B Biol Sci 354:1675-1686

4. Brooks WM, Wesley MH, Kodituwakku PW, Garry PJ, Rosenberg GA (1997) 1H-MRS differentiates white matter hyperintensities in subcortical arteriosclerotic encephalopathy from those in normal elderly. Stroke 28:1940-1943

5. Chabriat H, Bousser MG, Pappata S (1995) Cerebral autosomal dominant arteriopathy with subcortical infarcts and leukoencephalopathy: a positron emission tomography study in two affected family members. Stroke 26:1729-1730

6. Chabriat H, Pappata S, Poupon C, Clark CA, Vahedi K, Poupon F, Mangin JF, Pachot-Clouard M, Jobert A, Le Bihan D, Bousser MG (1999) Clinical severity in CADASIL related to ultrastructural damage in white matter: in vivo study with diffusion tensor MRI. Stroke 30:2637-2643

7. De Graaf RG (2007) In vivo NMR spectroscopy—principles and techniques, 2nd edn. Wiley, West Sussex

8. Dichgans M (2002) Cerebral autosomal dominant arteriopathy with subcortical infarcts and leukoencephalopathy: phenotypic and mutational spectrum. J Neurological Sci 203-204:77-80

9. Dichgans M (2007) Genetics of ischemic stroke. Lancet Neurol 6:149-161

10. Dichgans M, Mayer M, Uttner I, Bruening R, Mueller-Hoecker J, Rungger G, Ebke M, Klockgether T, Gasser T (1998) The phenotypic spectrum of CADASIL: clinical findings in 102 cases. Ann Neurol 44:731-739

11. Gray F, Polivka M, Viswanathan A, Baudrimont M, Bousser MG, Chabriat H (2007) Apoptosis in cerebral autosomal-dominant arteriopathy with subcortical infarcts and leukoencephalopathy. J Neuropathol Exp Neurol 66:597-607

12. Haase A, Frahm J, Hanicke W, Matthaei D (1985) 1H NMR chemical shift selective (CHESS) imaging. Phys Med Biol 30(4): 341-344

13. Henning A, Schär M, Schulte RF, Wilm B, Pruessmann KP, Boesiger P (2008) SELOVS: brain MRSI localization based on highly selective $\mathrm{T}_{1}$ - and $\mathrm{B}_{1}$-insensitive outer-volume suppression at 3T. Magn Reson Med 59:40-51

14. Joutel A, Corpechot C, Ducros A, Vahedi K, Chabriat H, Mouton P, Alamowitch S, Domenga V, Cécillion M, Marechal E, Maciazek J, Vayssiere C, Cruaud C, Cabanis EA, Ruchoux MM, Weissenbach J, Bach JF, Bousser MG, Tournier-Lasserve E (1996) Notch3 mutations in CADASIL, a hereditary adult-onset condition causing stroke and dementia. Nature 24:707-710

15. Jouvent E, Viswanathan A, Mangin JF, O'Sullivan M, Guichard JP, Gschwendtner A, Cumurciuc R, Buffon F, Peters N, Pachaï C, Bousser MG, Dichgans M, Chabriat H (2007) Brain atrophy is related to lacunar lesions and tissue microstructural changes in CADASIL. Stroke 38:1786-1790

16. Jung HH, Bassetti C, Tournier-Lasserve E, Vahedi K, Arnaboldi M, Arifi VB, Burgunder JM (1995) Cerebral autosomal dominant arteriopathy with subcortical infarcts and leukoencephalopathy: a clinicopathological and genetic study of a Swiss family. J Neurol Neurosurg Psychiatry 59:138-143

17. Kemp GJ (2000) Non-invasive methods for studying brain energy metabolism: what they show and what it means. Dev Neurosci 22:418-428

18. Lacombe P, Oligo C, Domenga V, Tournier-Lasserve E, Joutel A (2005) Impaired cerebral vasoreactivity in a transgenic mouse model of cerebral autosomal dominant arteriopathy with 
subcortical infarcts and leukoencephalopathy arteriopathy. Stroke 36:1053-1058

19. Macri MA, Colonnese C, Garreffa G, Fattapposta F, Restuccia R, Bianco F, Labruna L, Maraviglia B (2006) A chemical shift imaging study on regional metabolite distribution in a CADASIL family. Magn Reson Imaging 24:443-447

20. Markus HS, Martin RJ, Simpson MA, Dong YB, Ali N, Crosby AH, Powell JF (2002) Diagnostic strategies in CADASIL. Neurology 59:1134-1138

21. Mellies JK, Bäumer T, Müller JA, Tournier-Lasserve E, Chabriat H, Knobloch O, Hackelöer HJ, Goebel HH, Wetzig L, Haller P (1998) SPECT study of a German CADASIL family: a phenotype with migraine and progressive dementia only. Neurology 50:1715-1721

22. Oliveri RL, Mazzei R, Gabriele AL, Gambardella A (2001) A novel mutation in the Notch3 gene in an Italian family with cerebral autosomal dominant arteriopathy with subcortical infarcts and leukoencephalopathy - genetic and magnetic resonance spectroscopic findings. Arch Neurol 58:1418-1422

23. Pfefferkorn T, von Stuckrad-Barre S, Herzog J, Gasser T, Hamann GF, Dichgans M (2001) Reduced cerebrovascular CO(2) reactivity in CADASIL: a transcranial Doppler sonography study. Stroke 32:17-21

24. Provencher SW (1993) Estimation of metabolite concentrations from localized in vivo proton NMR spectra. Magn Reson Med 30:672-679
25. Reddy H, De Stefano N, Mortilla M, Federico A, Matthews PM (2002) Functional reorganization of motor cortex increases with greater axonal injury from CADASIL. Stroke 33:502-508

26. Rubio A, Rifkin D, Powers JM, Patel U, Stewart J, Faust P, Goldman JE, Mohr JP, Numaguchi Y, Jensen K (1997) Phenotypic variability of CADASIL and novel morphologic findings. Acta Neuropathol 94:247-254

27. Ruchoux MM, Chabriat H, Bousser MG, Baudrimont $M$, Tournier-Lasserve E (1994) Presence of ultrastructural arterial lesions in muscle and skin vessels of patients with CADASIL. Stroke 25:2291-2292

28. Ruchoux MM, Guerouaou D, Vandenhaute B, Pruvo JP, Vermersch P, Leys D (1995) Systemic vascular smooth muscle cell impairment in cerebral autosomal dominant arteriopathy with subcortical infarcts and leukoencephalopathy. Acta Neuropathol 89:500-512

29. Tournier-Lasserve E, Joutel A, Melki J, Weissenbach J, Lathrop GM, Chabriat H, Mas JL, Cabanis EA, Baudrimont M, Maciazek J, Bach MA, Bousser MG (1993) Cerebral autosomal dominant arteriopathy with subcortical infarcts and leukoencephalopathy maps to chromosome 19q12. Nat Genet 3:256-259

30. Walter M, Henning A, Grimm S, Schulte RF, Beck J, Dydak U, Schnepf B, Boeker H, Boesiger P, Northoff G (2009) The relationship between aberrant neuronal activation in the pregenual anterior cingulate, altered glutamatergic metabolism, and anhedonia in major depression. Arch Gen Psychiatry 66(5):478-486 